\title{
Thermal Modulation of Plasmon Induced Transparency in Graphene Metamaterial
}

Yuanlin Jia, Peiwen Ren, Junqiao Wang, Chunzhen Fan* and Erjun Liang

In order to obtain a dynamically adjustable plasmon induced transparency (PIT) phenomenon in non-contact way, we theoretically and numerically investigate the thermal tunable PIT by taking into account of electrons and phonons interaction in graphene metamaterial. The novel structure consists of two hollow square graphene and a rectangular strip in right middle, each of them acting as a bright mode. To fully explore the physical origin of the transparency window, the Lorentz coupled oscillator theory is employed. Good agreement can be achieved with the numerical results, which further verifies our calculations. To alter the PIT effect, the influence on the transparency window with different temperature and polarization angle are elaborately examined. The bandwidth of the transparent window gets wider with the lower temperature and switchable transparent window appears with varied polarization angle. As a potential application in the slow light device, the group index and group delay are also calculated with the temperature from $60 \mathrm{~K}$ to $500 \mathrm{~K}$. Our proposed thermal tunable PIT structure may open up a new avenue in the application of controllable optical filters, switchers and optical memories.

Keywords: Thermal modulation; Graphene metamaterial; Plasmon induced transparency

Received 25 September 2019, Accepted 21 October 2019

DOI: $10.30919 /$ esee8c338

\section{Introduction}

Metamaterials are engineered composite embodying some extraordinary physical properties beyond natural materials. The first experimental discovery of negative refraction was realized with metal metamaterials in $2000 .{ }^{1}$ Since then, it has rapidly become a hot topic among the researchers in optical, acoustic or thermal regime. The broad application prospects of metamaterials extend into the scope of superresolution imaging, ${ }^{2}$ electromagnetic stealth cloak, ${ }^{3}$ thermal cloak, ${ }^{4}$ thermal camouflage, ${ }^{5}$ acoustic lens ${ }^{6}$ and so on. One outstanding behavior in the optical region is the plasmon induced transparency (PIT), which is an analogue quantum interference effect in classical optical systems. ${ }^{7,8}$ PIT was firstly proposed by Zhang et al. in 2008, which based on metal metamaterials composing of three silver strips. ${ }^{9}$ The mutual coupling between the bright and dark mode resulted in a narrow band transparent window in the transmission spectrum. With the maturity of plasmon photonics and metamaterial fabrication technology, PIT has been intensively investigated in the area of flexible design, adjustable working bands and simple implementation conditions. It has wide practical applications in optical sensors, filters, absorbers and switchers. ${ }^{10-13}$ However, metal metamaterials have some intrinsic drawbacks to get tunable PIT, like the large propagation loss and high cost, especially the resonant peak and operating frequency band are not able to flexibly tune once the structures are fabricated.

The emergence of new materials like the graphene, ${ }^{14,15}$ Dirac semimetal $^{16}$ and phase change materials ${ }^{17}$ provide us a new way to get tunable PIT effect, owing to their outstanding electrical, thermal and optical properties. In the modulation process, the controllable PIT can be obtained by varying the Fermi energy, ${ }^{18}$ the polarization angle ${ }^{19}$ or the thermal stimuli. ${ }^{20}$ Jia et al. investigated the active control and large group delay in graphene based $\mathrm{THz}$ metamaterial with external applied gate voltage. ${ }^{21}$ Liu et al. designed H-shaped Dirac semimetal metamaterial to achieve thermal modulation of PIT during the process of rising temperature. ${ }^{20} \mathrm{~A}$ phase change material $\mathrm{VO}_{2}$ 
combined with SRR metamaterials had been fabricated to achieve tuning transmission spectrum by thermal modulation. ${ }^{22}$ Kurter et al. experimentally investigated the transparency window employing a superconducting $\mathrm{Nb}$ film and $\mathrm{Au}$ film below the critical temperature of $\mathrm{Nb}^{23}$ Temperature controlling of the metamaterials comprising a pair of gold strips and a vanadium dioxide strip had also been achieved in Ref.17. In addition, thermal transparency was theoretically investigated with an effective medium theory by considering anisotropic layered or graded structures. ${ }^{24}$ This phenomenon was realized by tailoring periodic interparticle interaction, enhancing the flexibility of thermal management. ${ }^{25}$ Cao et al. experimentally observed the thermal modulation of PIT effect with superconductor-metal coupled resonator. ${ }^{26} \mathrm{Li}$ et al. realized the thermal modulation of PIT in the waveguide structure by using the plasma frequency of InSb. ${ }^{27}$ Therefore, research on thermal modulation of PIT in graphene metamaterial is currently very rare. Combining the external thermal modulation and the outstanding properties of graphene metamaterials, the potential applications can extend into the area of thermal filters, thermal rectifiers, ${ }^{28}$ and thermal emitters. ${ }^{29}$ Moreover, thermal modulation method is convenient to initiate in the experiment.

In this work, we have proposed the patterned graphene metamaterial to achieve tunable PIT effect through thermal management with the Boltzmann transport theory. Owing to the near field coupling between the two bright modes, a clear transparent window is created, which is further verified by the Lorentz coupled oscillator theory. The bandwidth and intensity of the PIT transparency window are fully explored with the temperature from $60 \mathrm{~K}$ to $500 \mathrm{~K}$. Moreover, the number of transparent windows can be tuned by adjusting the polarization angle. Finally, the dispersion properties in our proposed structure are investigated with the calculation of group index and delay time.

\section{Theory and Model}

\subsection{Boltzmann Transport Theory Includes the Electron- Phonon Scattering in Graphene}

We consider the electron-phonon interaction in the graphene metamaterial under the influence of thermal stimuli with Boltzmann transport theory. The contribution of phonon modes to carrier transport under different thermal range is fully considered. There are six phonon branches in graphene, three optical phonon branches and three acoustic phonon branches. ${ }^{30,31}$ When the temperature below $200 \mathrm{~K}$, the longitudinal acoustic phonon scattering plays a primary role, while the optical phonon can' $t$ be excited. The number of optical phonons increases with the high temperature above 200 $\mathrm{K} .{ }^{32,33}$ Thus, taking the influence of thermal on the scattering rate between phonons and carriers into considerations, an integral differential equation describing how the electron distribution function $f(\boldsymbol{r}, \boldsymbol{k}, \mathrm{t})$ varies in the phase space is employed $^{34}$

$$
\begin{gathered}
\boldsymbol{v}_{\boldsymbol{k}} \cdot \nabla_{r} f(\boldsymbol{r}, \boldsymbol{k}, t)-\frac{e}{\hbar}(\boldsymbol{E}+\boldsymbol{v} \times \boldsymbol{B}) \cdot \nabla_{r} f(\boldsymbol{r}, \boldsymbol{k}, t) \\
-\sum_{k}\left\{W\left(\boldsymbol{k}^{\prime}, \boldsymbol{k}\right) f\left(\boldsymbol{k}^{\prime}\right)[1-f(\boldsymbol{k})]-W\left(\boldsymbol{k}, \boldsymbol{k}^{\prime}\right) f(\boldsymbol{k})\left[1-f\left(\boldsymbol{k}^{\prime}\right)\right]\right\}=0,
\end{gathered}
$$

$\boldsymbol{k}$ is the wave vector. The first term denotes the diffusion of electrons in real space, the second term represents the inverse space drift of electrons under the steady external field and the third term is related to electron scattering. $\boldsymbol{E}$ and $\boldsymbol{B}$ are the electric and magnetic fields in the external field respectively. $\boldsymbol{v}_{\boldsymbol{k}}$ is the group velocity of electrons and it equals to $\boldsymbol{v}_{\boldsymbol{k}}=\nabla_{k} \varepsilon_{k} /$ $\hbar . W\left(\boldsymbol{k}^{\prime}, \boldsymbol{k}\right)$ is the probability of an electron going from $\boldsymbol{k}^{\prime}$ to $\boldsymbol{k}$ state per unit time. $W\left(\boldsymbol{k}, \boldsymbol{k}^{\prime}\right)$ is the probability of the electron leaving the $\boldsymbol{k}$ state and going into the $\boldsymbol{k}^{\prime}$ state. Under the condition of quasi-elastic acoustic scattering, ${ }^{35,36} \mathrm{~W}\left(k^{\prime}, k\right)=$ $\mathrm{W}\left(k, k^{\prime}\right)=\frac{2 \pi}{\hbar} \sum|C(q)|^{2} \Delta\left(\varepsilon, \varepsilon^{\prime}\right) . \quad \varepsilon \quad$ and $\quad \varepsilon^{\prime}$ represent the energy of the $\boldsymbol{k}$ and $\boldsymbol{k}^{\prime}$ states of the electron respectively. $q$ is the phonon wave vector. $C(q)$ is acoustic phonon scattering matrix element and taken as $|C(q)|^{2}=$ $D^{2} \hbar q\left[1-\left(\frac{q}{2 k}\right)^{2}\right] / 2 A \rho_{m} v_{p h}, \quad \Delta\left(\varepsilon, \varepsilon^{\prime}\right)=N_{\mathrm{q}} \delta\left(\varepsilon-\varepsilon^{\prime}+\hbar \omega_{\mathrm{q}}\right)+$ $\left(N_{q}+1\right) \delta\left(\varepsilon-\varepsilon^{\prime}-\hbar \omega_{q}\right)$, where $N_{q}$ is the phonon occupation number. It can be written as $N_{q}=1 /\left(\exp \left(\hbar \omega_{q} / k_{B} T\right)-1\right)$. $D$ is the deformation potential coupling constant. $\rho_{m}$ is the graphene mass density, $\omega_{q}=v_{p h} q$ represents the acoustic phonon energy with the phonon velocity. A is the area of the sample. Under the relaxation time approximation, the scattering rate of electron and acoustic phonons in the intraband can be indicated as: ${ }^{36}$

$$
\frac{1}{\tau_{a c, k k}}=\frac{E_{F} D^{2} k_{B} T}{4 \hbar^{3} v_{F}^{2} \rho_{m} v_{p h}^{2}} .
$$

The electron scattering rate incorporating the optical phonons and acoustic phonons in the interband can be written as: $: 37$

$$
\begin{aligned}
& \frac{1}{\tau_{o p}}= \\
& \frac{D_{0}^{2}}{\rho_{m} \omega_{0}\left(\hbar v_{F}\right)^{2}}\left[\left(E_{F}-\hbar \omega_{0}\right)\left(N_{q}+1\right) \Theta\left(E_{F}-\hbar \omega_{0}\right)+\left(E_{F}+\hbar \omega_{0}\right) N_{q}\right],
\end{aligned}
$$

$\mathrm{D}_{0}$ is the optical phonon deformation field. $\omega_{0}$ is the optical phonon frequency, $\Theta$ is the Heaviside function. The total scattering rate can be obtained as: $:^{32}$

$$
\frac{1}{\tau}=\frac{1}{\tau_{a c, k k}}+\frac{1}{\tau_{a c, k k^{\prime}}}+\frac{1}{\tau_{o p}} .
$$

Moreover, the conductivity of graphene can be obtained with Kubo formula, ${ }^{38}$ which consists the contribution of interband and intra-band under the excitation. It can be denoted as,

$$
\sigma\left(\omega, \tau, \mathrm{E}_{\mathrm{F}} \mathrm{T}\right)=\frac{i e^{2}}{\pi \hbar^{2}\left(\omega+i \hbar \tau^{-1}\right)} \int_{0}^{\infty} \xi\left(\frac{\partial f_{\mathrm{d}}(\xi)}{\partial \xi}-\frac{\partial f_{\mathrm{d}}(-\xi)}{\partial \xi}\right) d \xi+
$$




$$
\frac{i e^{2}\left(\omega+i \hbar \tau^{-1}\right)}{\pi \hbar^{2}} \int_{0}^{\infty} \frac{f_{d}(-\xi)-f_{d}(\xi)}{\left(\omega+i \hbar \tau^{-1}\right)^{2}-4(\xi / \hbar)^{2}} d \xi .
$$

$\omega, \tau, E_{F}$ and $T$ represent frequency, relaxation time, Fermi energy and temperature, respectively. $\mathrm{f}_{d}(\xi)=$ $1 /\left(\exp \left(\left(\xi-E_{F}\right) /\left(k_{B} T\right)\right)+1\right)$ is the Fermi-Dirac distribution, $e$ is the electronic charge. $\hbar$ denotes reduced Planck constant. $k_{B}$ represents the Boltzmann constant. The relationship between the real part of the conductivity and temperature is illustrated in Fig. 1. It can be clearly observed that the real part of the conductivity increases with higher temperature in a linear way. Thus, it is necessary to study the electron-phonon interaction with different thermal excitation.

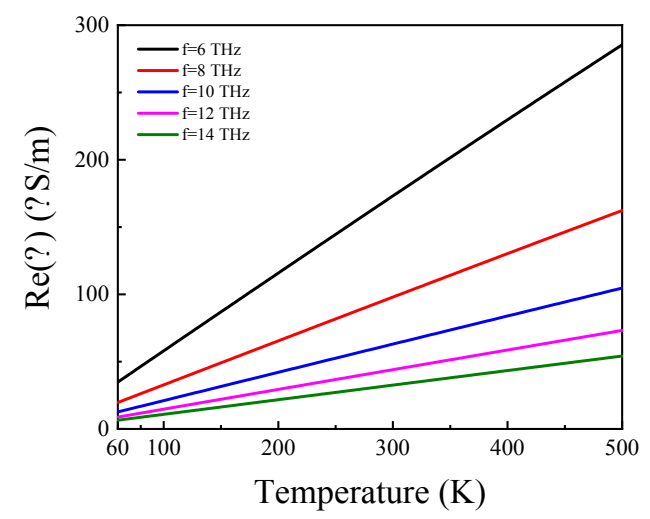

Fig. 1 The relationship between the real part of the graphene conductivity and temperature is calculated under certain excitation frequency.

\subsection{Model Design}

Our designed graphene metamaterial is shown in Fig. 2. The patterned graphene layer is periodically deposited on the dielectric substrate (silicon) in Fig. 2a. Its thickness is $200 \mathrm{~nm}$. The incident light is along $z$ direction and the polarized electric field is along $x$ axis. The unit cell consisting of two hollow square graphene and a rectangular graphene strip is depicted in Fig. 2b. The periodicity along the $x$ and $y$ axis is $P_{x}=P_{y}=$ $600 \mathrm{~nm}$. The length and width of the graphene strip are $l_{1}=450$ $\mathrm{nm}$ and $w_{1}=110 \mathrm{~nm}$. Inner and outer length of the square graphene are $l_{3}=110 \mathrm{~nm}$ and $l_{2}=150 \mathrm{~nm}$. The distance between the strip and square is $d$, which is fixed at $20 \mathrm{~nm}$. In our numerically calculation, one-unit cell with periodicity boundary is considered for simplicity. The Fermi energy of the graphene is taken as $0.5 \mathrm{eV}$ in our numerical calculations. Up to now, there are several methods to prepare the single layer graphene like the mechanical exfoliation method, ${ }^{39}$ epitaxial growth method, ${ }^{40}$ oxidation-reduction method ${ }^{41}$ and chemical vapor deposition (CVD) ${ }^{42}$ In order to produce large-scale, highquality graphene layer at low-cost, CVD is the best choice to obtain the graphene on the $\mathrm{Cu}$ substrate, then it can be transferred onto the dielectric substrate. After that, electron beam lithography and anisotropic plasma etching can be employed to prepare patterned graphene films. ${ }^{43}$

\section{Results and Discussions}

The transmission spectrum of the incident light propagating through the graphene metamaterial at $60 \mathrm{~K}$ are illustrated in Fig. 3a. A broadband transparency window at $10.2 \mathrm{THz}$ can be achieved in the transmission spectra (black line) in our proposed structure. As a comparison, we have also investigated the transmission spectra in each separate resonator. A dip locating at $9.1 \mathrm{THz}$ can be clearly achieved in the structure of only horizontal graphene strip, indicating a directive interaction with the incident light, acting as a bright mode. In the case of two symmetrical hollow square graphene (blue line), a dip around $11.3 \mathrm{THz}$ exists. To fully explore the physical origin of the resonant peaks, the electric field distribution at resonant position of $9.15 \mathrm{THz}$, (a)

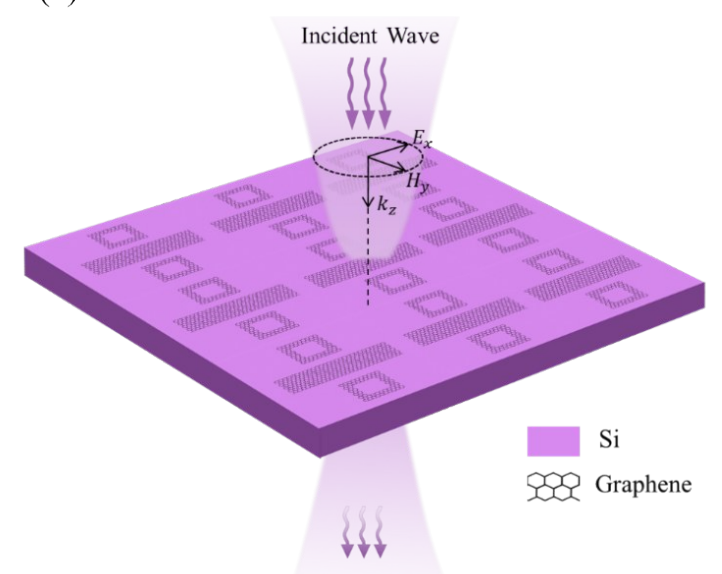

(b)

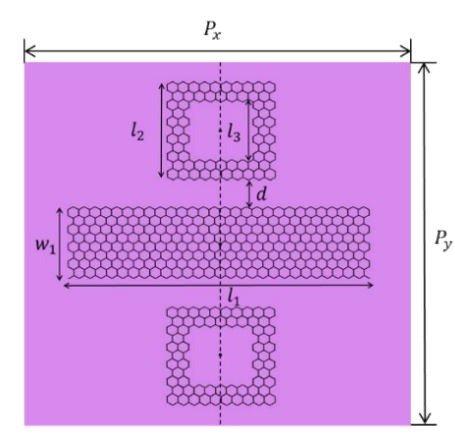

Fig. 2 (a) Schematic diagram of the patterned graphene metamaterial. (b) Top view of the unit cell with geometric parameters; $w_{1}=110 \mathrm{~nm} ; l_{1}=450 \mathrm{~nm} ; l_{2}=150 \mathrm{~nm} ; l_{3}=110$ $n m ; P_{x}=P_{y}=600 \mathrm{~nm} ; d=20 \mathrm{~nm}$. 

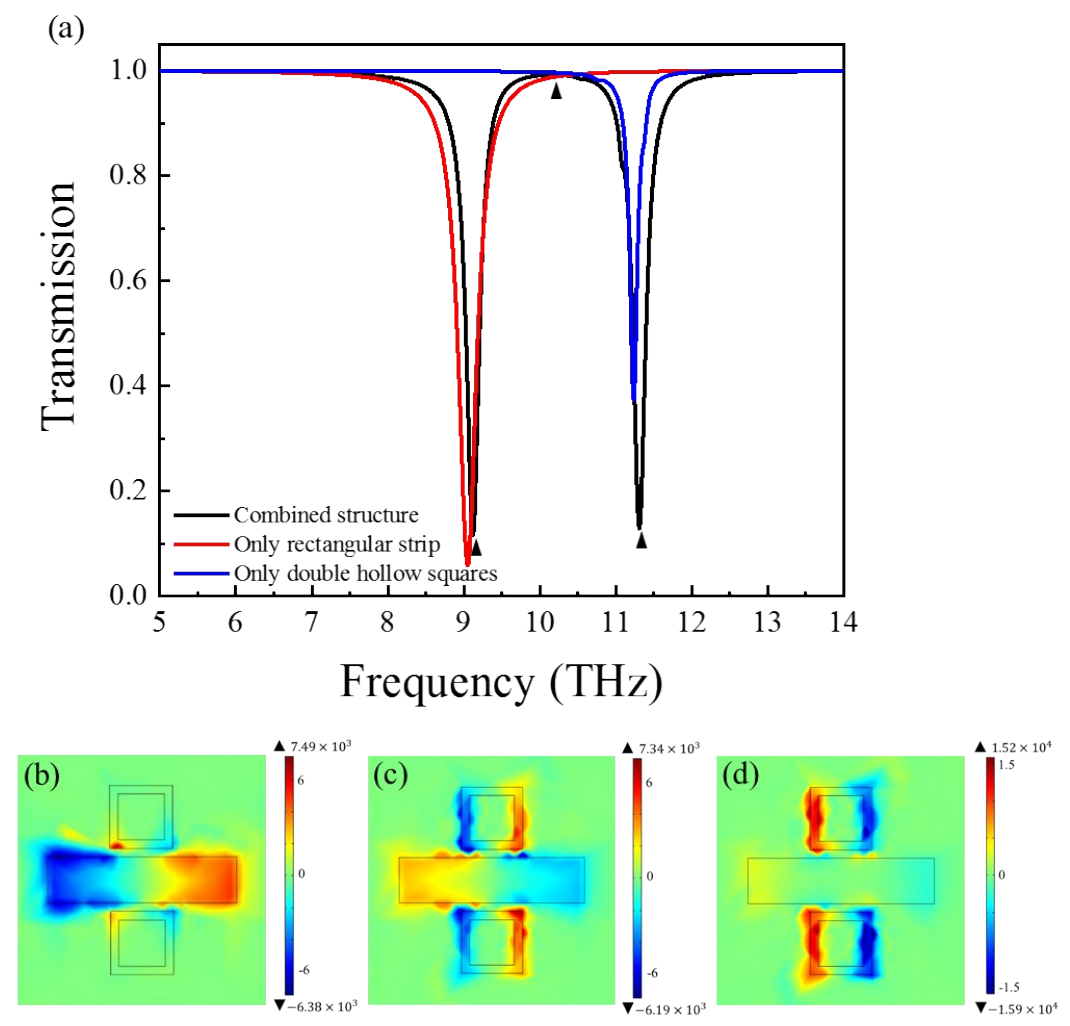

Fig. 3 (a) The transmission spectra of the patterned graphene metamaterial are investigated for the only rectangular strip, only double hollow squares and combined structure at $60 \mathrm{~K}$, respectively. (b-d) The electric field distribution at each resonant position is shown.

10.2 THz and 11.3 THz are also investigated in Figs. 3(b-d). The electric field is mainly distributed on the two sides of the graphene strip, behaving as a dipole resonator in Fig. 3b. Similarly, the electric filed in the double square resonators mainly focuses on the left and right sides in Fig. $3 \mathrm{~d}$. For the combined structure, due to the near filed coupling between them, the electric field is distributed in each resonator in Fig. 3c. Thus, an obvious broadband transparency window can be achieved in the transmission spectra. The exact position and intensity of the plasmon resonant peak is closely related to the geometry parameters (like size and shape) and its medium surrounding medium. ${ }^{44}$ With our proposed graphene structure, the PIT transparent window can be well regulated into the mid-infrared region through well-chosen size and Fermi energy.

Thermal tunable broadband PIT effect is also explored in Fig. 4a. With the increasing temperature from $60 \mathrm{~K}$ to $500 \mathrm{~K}$, there is no shift of PIT transparency window position, whereas the intensity and bandwidth of transparent window are decreasing. Namely, owing to the weak interaction of electron and phonon scattering rate and reduced thermal loss, larger bandwidth of the transmission window at lower temperature can be obtained. With the Lorentz coupled oscillator theory, ${ }^{45}$ the validation of our numerically results is carried out. Under the excitation of the incident light $E_{0} e^{i \omega t}$, the bright mode of the strip and squares resonator can be treated as an oscillator and it can be described as $|a\rangle=\tilde{a}(\omega) e^{i \omega t}$ and $|b\rangle=\tilde{b}(\omega) e^{i \omega t}$. The amplitude of two bright modes incorporating their mutual interaction can be depicted as following,

$$
\left(\begin{array}{cc}
\omega-\omega_{1}+i \gamma_{1} & \kappa \\
\kappa & \omega-\omega_{2}+i \gamma_{2}
\end{array}\right)\left(\begin{array}{l}
\tilde{a} \\
\tilde{b}
\end{array}\right)=\left(\begin{array}{l}
g_{1} \tilde{E}_{0} \\
g_{2} \\
\tilde{E}_{0}
\end{array}\right),
$$

$\omega_{i}, \gamma_{i}$ and $g_{i}$ represent the resonant frequency, damping factor and coupling strength respectively. $\kappa$ is coupling strength between two bright modes. The transmission spectrum of the proposed PIT structure can be represented as $T=1-\left|\tilde{a} / \tilde{E}_{0}\right|^{2}$. The calculated transmission spectra under different temperature with the Lorentz coupled oscillator theory is presented in Fig. 4b. It can be clearly observed that these two results fit well with each other, which fully verifies the correctness of our numerical results. Moreover, the fitting parameters at different temperatures are elaborately shown in Table 1. The damping factor $\gamma$ is an important parameter in the harmonic oscillator equations, which measures the rate of oscillation when the incident plane wave is removed. The damping factors $\gamma_{1}$ and $\gamma_{2}$ of two bright modes increase with a higher temperature, indicating that the autologous loss of graphene affects the 


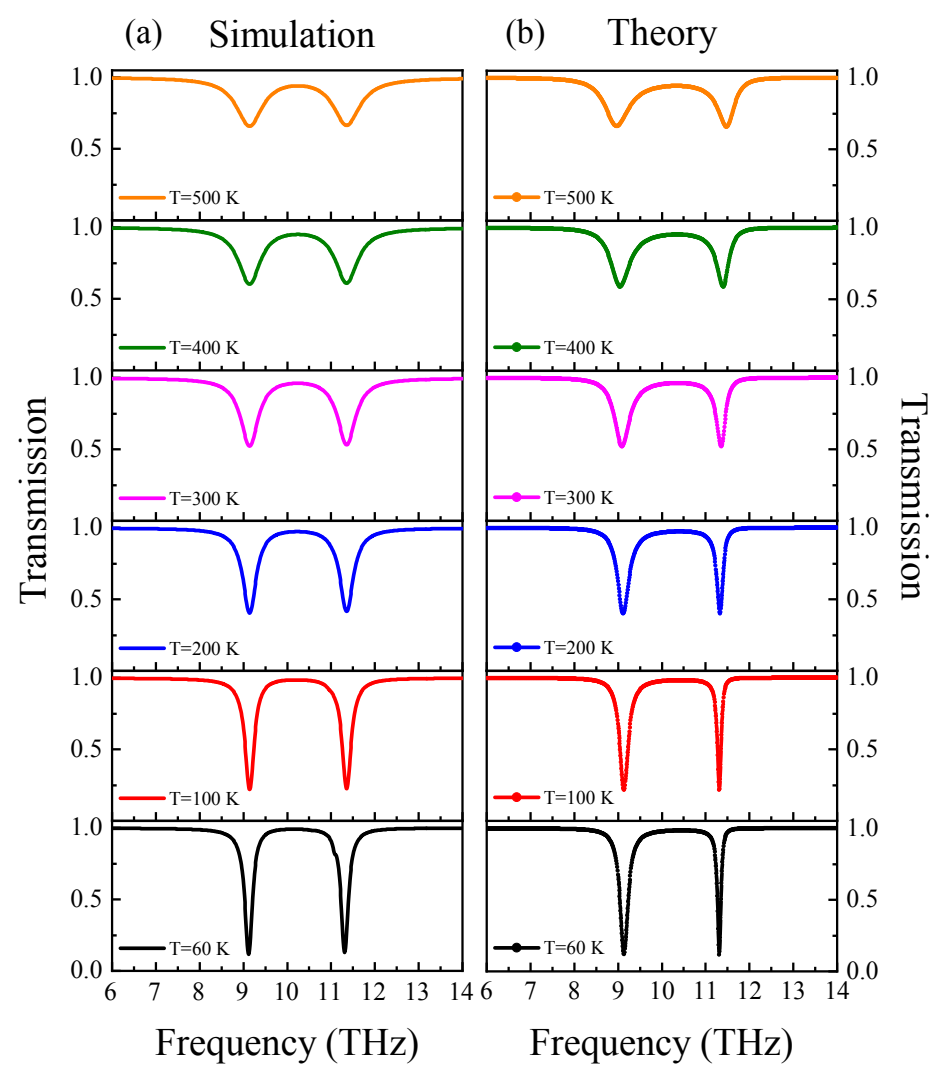

Fig. 4 (a) The transmission spectra with different temperature. (b) The fitted transmission spectra with the Lorentz coupled oscillator theory.

Table 1 Fitting parameters with the Lorentz coupled oscillator theory at different temperature are listed.

\begin{tabular}{cccccc}
\hline$T(\mathrm{~K})$ & $\Upsilon_{1}(\mathrm{THz})$ & $\Upsilon_{2}(\mathrm{THz})$ & $\kappa(\mathrm{THz})$ & $\omega_{1}(\mathrm{THz})$ & $\omega_{2}(\mathrm{THz})$ \\
\hline 60 & 0.108 & 0.043 & 0.18 & 9.15 & 11.3 \\
100 & 0.117 & 0.048 & 0.19 & 9.15 & 11.3 \\
200 & 0.159 & 0.079 & 0.28 & 9.15 & 11.3 \\
300 & 0.210 & 0.118 & 0.39 & 9.15 & 11.3 \\
400 & 0.264 & 0.160 & 0.52 & 9.15 & 11.3 \\
500 & 0.340 & 0.215 & 0.70 & 9.15 & 11.3 \\
\hline
\end{tabular}

damping coefficient of the two bright mode resonators. Another parameter $\kappa$ denotes the coupling coefficient between these two resonators and it also becomes larger with a higher temperature. Thus, the PIT transparency window is caused to happen through the weak coupling between these two resonators.

Moreover, we have also emphatically analyzed the tunable bandwidth of the transparency window with the temperature ranging from $60 \mathrm{~K}$ to $500 \mathrm{~K}$ in Fig. 5. The bandwidth at each temperature is extracted out and plotted in Fig. 5a. It can be clearly observed that with the higher thermal modulation, the bandwidth gets decreased. Namely, the band width ranges from $9.87 \mathrm{THz}$ to $10.54 \mathrm{THz}$ at $60 \mathrm{~K}$. It decreases from $10.54 \mathrm{THz}$ to $10.05 \mathrm{THz}$ at $500 \mathrm{~K}$. As a potential application of the optical filter, specific wavelength can be achieved with careful chosen thermal initiation. The resonant peak position of transmission peak also decreases linearly with increasing temperature in Fig. 5b. The transmittance is as high as 0.99 at $60 \mathrm{~K}$, but it decreases to 0.94 at $500 \mathrm{~K}$. The reduced number of acoustic phonons and optical phonons in graphene, the increased scattering rate of electron phonons, and enhanced thermal loss at higher temperature, result in the decreased transmittance intensity.

Two PIT transparency windows can be achieved in our proposed structure with the applied polarization angle, which is an effective tool to get tunable PIT phenomena. The transmission spectra at $60 \mathrm{~K}$ with different polarization 
(a)

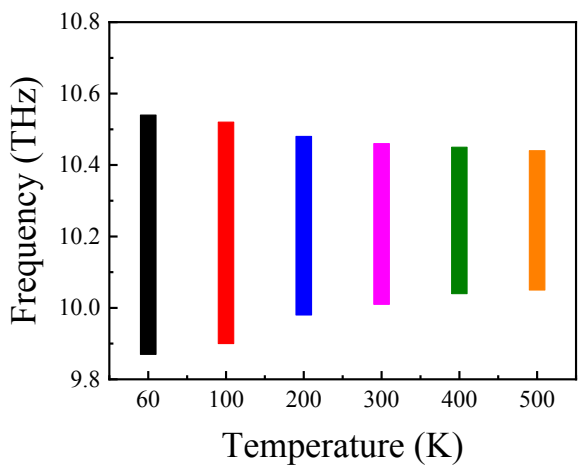

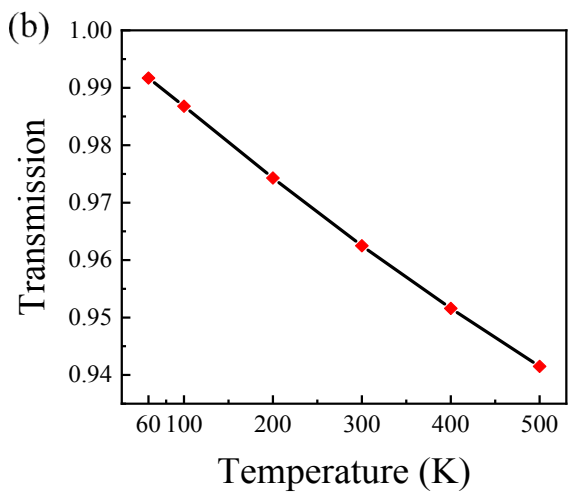

Fig. 5 (a) The bandwidth and (b) resonant peak position at different temperature are illustrated.

angle are shown in Fig. 6. $\theta$ is defined as the angle between the polarization direction and the $x$ direction. $\theta=0^{\circ}$ is the polarization of the electric field along the $x$ direction; $\theta=$ $90^{\circ}$ corresponds to the polarization of the electric field along the $y$ direction. When $\theta$ increases to $15^{\circ}$, the second PIT transparency comes to appear. It becomes obvious at $30^{\circ}$. The transmission spectrum transforms from one transparent window to two transparent windows with a larger polarization angle. Two PIT transparency windows can be clearly observed with $45^{\circ}$ polarization angle. The first PIT window is generated by the coupling between two bright modes. The second PIT window is caused by the asymmetric electric field distribution around the resonators. With a further increasing of the polarization angle, two transparent windows disappear and only one dip exists.

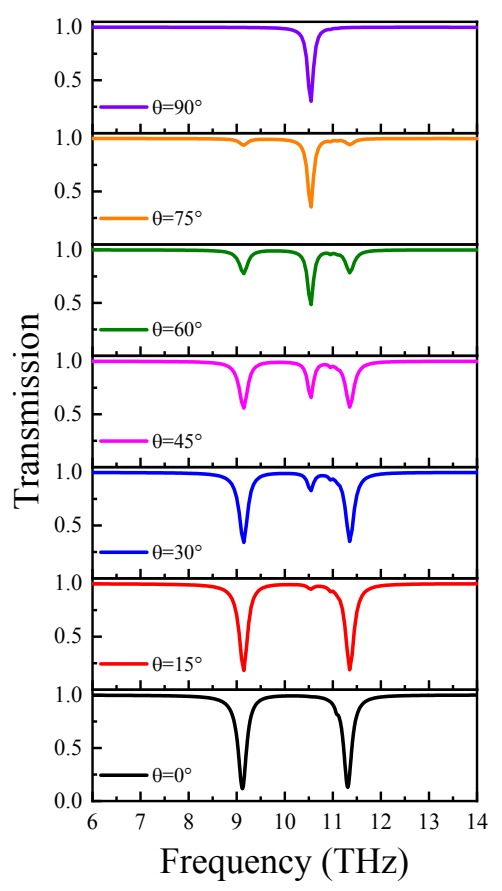

Fig. 6 Transmission spectra with different polarization angles is illustrated at $60 \mathrm{~K}$.

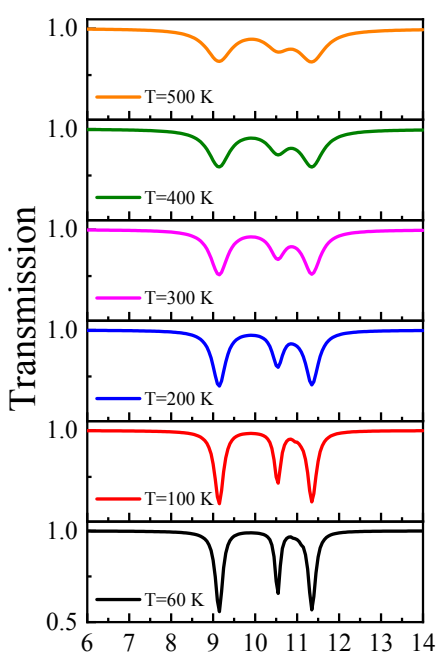

Frequency (THz)

Fig. 7 Transmission spectrum is calculated with different temperature at $45^{\circ}$ polarization angle.

Fig. 7 describes the transmittance in our proposed graphene metamaterials with $45^{\circ}$ polarization angle at different temperature. It is found that the intensity of two PIT window can be effectively tuned with thermal modulation. The higher temperature leads to the lower intensity of the PIT effect. Two transparent windows diminish with higher temperature due to the enhanced electron-phonon scattering rate and the thermal loss.

One striking feature of the PIT phenomenon is the slow light effect around transparent window, which can be reflected with the calculation of the group index $n_{g}=\frac{c}{t} \frac{d \varphi}{d \omega}$ and group delay $t_{g}=-\frac{d \varphi}{d \omega},{ }^{46}$ where $\varphi$ is the phase, $\omega$ is the angular frequency of incident light, $C$ is the speed of light, and $t_{s}$ is the thickness of substrate. Fig. 8 illustrates the group index and group delay time as a function of the temperature. With the higher temperature, the group index at the resonant position decreases gradually. It varies from 
2238 to 584 when the temperature is taken from $60 \mathrm{~K}$ to $500 \mathrm{~K}$. Meanwhile, the group delay varies from $1.45 \mathrm{ps}$ to $0.39 \mathrm{ps}$. It provides us an effective way in the application of optical memories.

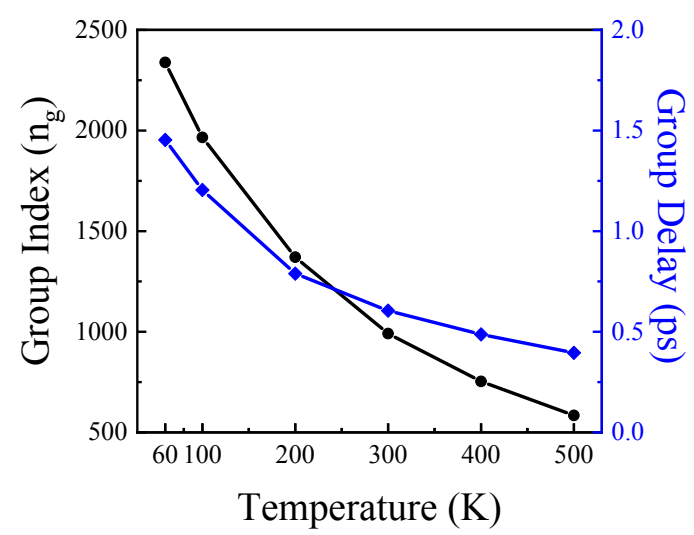

Fig. 8 Group index and group delay as a function of the temperature are illustrated.

\section{Conclusion}

In summary, thermal regulation of the PIT effect is realized in our proposed graphene metamaterial incorporating the electron and phonon scattering with Boltzmann transport theory. The PIT transparency window is caused to happen through the weak coupling between two resonators. Our numerical results coincide well with the Lorentz coupled oscillator theory. The bandwidth and resonant peak position can be effectively tuned with the initiation of different heat source. Moreover, the number of the PIT transparency window can be varied with the polarization angle. As in the application of slow light device, it is found that the maximum group index of 2338 and group delay of $1.45 \mathrm{ps}$ can be achieved at $60 \mathrm{~K}$. Therefore, our work will deliver some potential applications in the thermal tunable optical filters, sensors and slow light equipment.

\section{Conflict of interest}

There are no conflicts to declare.

\section{Acknowledgements}

We are grateful to the financial supports from the National Natural Science Foundation of China (No. 11874328) and the Key science and technology research project of Henan Province (No. 1721023100107).

\section{References}

1. D. R. Smith, W. J. Padilla, D. C. Vier, S. C. N. Nasser and S. Schultz, Phys. Rev. Lett., 2000, 84, 4184.

2. J. B. Pendry, Phys. Rev. Lett., 2000, 85, 3966.

3. J. B. Pendry, D. Schurig and D. R. Smith, Science, 2006, 312, 17801782 .

4. C. Z. Fan, Y. Gao and J. P. Huang, Appl. Phys. Lett., 2008, 92, 251907.
5. R. Z. Wang, J. Shang and J. P. Huang, Int. J. Therm. Sci., 2018, 131, 1419.

6. W. Q. Wang, Y. B. Xie, A. Konneker, B. I. Popa and S. A. Cummer, Appl. Phys. Lett., 2014, 105, 101904.

7. K. J. Boller, A. Imamoğlu and S. E. Harris, Phys. Rev. Lett., 1991, 66, 2593.

8. B. Luk'yanchuk, N. I. Zheludev, S. A. Maier, N. J. Halas, P. J. Nordlander, H. Giessen and C. T. Chong, Nat. Mater, 2010, 9, 707.

9. S. Zhang, D. A. Genov, Y. Wang, M. Liu and X. Zhang, Phys. Rev. Lett., 2008, 101, 047401.

10. N. Liu, T. Weiss, M. Mesch, L. Langguth, U. Eigenthaler, M. Hirscher, C. Sönnichsen and H. Giessen, Nano Lett., 2009, 10, 1103-1107.

11. Z. Gao, Dong, H. Liu, J. X. Cao, T. Li, S. M. Wang, S. N. Zhu and X. Zhang. Appl. Phys. Lett., 2010, 97, 114101.

12. J. Chen, P. Wang, C. Chen, Y. Lu, H. Ming and Q. Zhan, Opt. Express, 2011, 19, 5970-5978.

13. J. N. Zhang, G. C. Wang, B. Zhang, T. He, Y. A. He and J. L. Shen, Opt. Mater., 2016, 54, 32-36.

14. K. S. Novoselov, V. I. Fal' ko, L. Colombo, P. R. Gellert, M. G. Schwab and K. Kim, Nature, 2012, 490, 192.

15. C. Liu, M. Chen, W. Yu and Y. L. He, ES Energy Environ., 2018, 2, 31 42 .

16. O. V. Kotov and Y. E. Lozovik, Phys. Rev. B, 2016, 93, 235417.

17. W. X. Huang, X. G. Yin, C. P. Huang, Q. J. Wang, T. F. Miao and Y. Y. Zhu, Appl. Phys. Lett., 2010, 96, 261908.

18. P. W. Ren, Y. L. Jia, W. Jia and C. Z. Fan, J. Opt., 2019, 21, 105101

19. Y. H. Ling, L. R. Huang, W. Hong, T. J. Liu, J. Luan, W. B. Liu, J. J. Lai and H. P. Li, Nanoscale, 2018, 10, 19517-19523.

20. Y. Liu, Y. Du, W. Liu, S. Shen, Q. Tan, J. Xiong and W. Zhang, Plasmonics, 2019, 1-7.

21. W. Jia, P. W. Ren, Y. L. Jia and C. Z. Fan, J. Phys. Chem. C., 2019, 123, 18560-18564.

22. T. Driscoll, S. Palit, M. M. Qazilbash, M. Brehm, F. Keilmann, B. G. Chae, S. J. Yun, H. T. Kim, S. Y. Cho, N. M. Jokerst, D. R. Smith and D. N. Basov, Appl. Phys. Lett., 2008, 93, 024101.

23. C. Kurter, P. Tassin, L. Zhang, T. Koschny, A. P. Zhuravel, A. V. Ustinov, S. M. Anlage and C. M. Soukoulis, Phys. Rev. Lett., 2011, 107, 043901 .

24. R. Z. Wang, L. J. Xu, Q. Ji and J. P. Huang, J. Appl. Phys., 2018, 123, 115117.

25. L. J. Xu, S. Yang and J. P. Huang, Phys. Rev. Appl., 2019, 11, 034056

26. W. Cao, R. J. Singh, C. H. Zhang, J. G. Han, M. Tonouchi and W. L. Zhang, Appl. Phys. Lett., 2013, 103, 101106.

27. Y. Li, Y. Su, X. Zhai and L. L. Wang, EPL-Europhys Lett, 2019, 125, 34002.

28. Z. H. Zheng, X. L. Liu, A. Wang and Y. M. Xuan, Int. J. Heat. Mass. Tran., 2017, 109, 63-72.

29. C. Shi, N. H. Mahlmeister, I. J. Luxmoore, and G. R. Nash, Nano. Res., 2018, 11, 3567-3573.

30. A. Alofi and G. P. Srivastava, J. Appl. Phys., 2012, 112, 013517.

31. H. Bao, J. Chen, X. Gu and B. Cao, ES Energy Environ., 2018, 1, 16-55.

32. K. M. Borysenko, J. T. Mullen, E. A. Barry, S. Paul, Y. G. Semenov, J. M. Zavada, M. B. Nardelli and K. W. Kim, Phys. Rev. B, 2010, 81, 121412.

33. Z. X. Lu and X. L. Ruan, ES Energy Environ. 2019, 4, 5-14.

34. J. M. Ziman, Principles of the Theory of Solids, Cambridge university press, 1979

35. J. Bardeen and W. Shockley, Phys. Rev., 1950, 80, 72.

36. E. H. Hwang and S. D. Sarma, Phys. Rev. B, 2008, 77, 115449.

37. R. S. Shishir and D. K. Ferry, J. Phys.: Condens. Matter, 2009, 21, 344201.

38. G. W. Hanson, J. Appl. Phys., 2008, 103, 064302

39. K. S. Novoselov, A. K. Geim, S. V. Morozov, D. Jiang, Y. Zhang, S. V. 
Dubonos, I. V. Grigorieva and A. A. Firsov, Science, 2004, 306, 666-669.

40. C. Berger, Z. M. Song, X. B. Li, X. S. Wu, N. Brown, C. Naud, D. Mayou, T. B. Li, J. Hass, A. N. Marchenkov, E. H. Conrad, P. N. First and W. A. D. Heer, Science, 2006, 312, 1191.

41. S. Stankovich1, D. A. Dikin, G. H. B. Dommett, K. M. Kohlhaas, E. J. Zimney, E. A. Stach, R. D. Piner, S. T. Nguyen and R. S. Ruoff, Nature, 2006, 442, 282-286.

42. A.N. Obraztsov, Nat. Nanotechnol., 2009, 4, 212.

43. R. Yang, L. C. Zhang, Y. Wang, Z. W. Shi, D. X. Shi, H. J. Gao, E. N.
Wang and G. Y. Zhang, Adv. Mater., 2010, 22, 4014-4019.

44. L. M. Liz-Marzán and P. Mulvaney, New J. Chem., 1998, 22, 1285-1288.

45. X. Shang, X. Zhai, X. Li, L. Wang, B. Wang and G. Liu, Plasmonics, 2016, 11, 419-423.

46. H. Lu, X. Liu and D. Mao, Phys. Rev. A, 2012, 85, 053803.

Publisher's Note Engineered Science Publisher remains neutral with regard to jurisdictional claims in published maps and institutional affiliations. 